\title{
Learning Similarity Measure of Nominal Features in CBR Classifiers
}

\author{
Yan $\mathrm{Li}^{1}$, Simon Chi-Keung Shiu ${ }^{1}$, Sankar Kumar Pal ${ }^{2}$, and James Nga-Kwok Liu ${ }^{1}$ \\ ${ }^{1}$ Department of Computing, Hong Kong Polytechnic University, \\ Kowloon, HongKong \\ ${ }^{2}$ Machine Intelligence Unit, Indian Statistical Institute, \\ Kolkata, 700 035, India \\ \{csyli, csckshiu, csnkliu\}@comp.polyu.edu.hk, \\ sankar@isical.ac.in
}

\begin{abstract}
Nominal feature is one type of symbolic features, whose feature values are completely unordered. The most often used existing similarity metrics for symbolic features is the Hamming metric, where similarity computation is coarse-grained and may affect the performance of case retrieval and then the classification accuracy. This paper presents a GA-based approach for learning similarity measure of nominal features for CBR classifiers. Based on the learned similarities, the classification accuracy can be improved, and the importance of each nominal feature can be analyzed to enhance the understanding of the used data sets.
\end{abstract}

\section{Introduction}

In the past decades, CBR systems [1, 2] have been successfully applied to various domains, among which those used in classification problems are called CBR classifiers. Since the basic assumption in CBR is that similar problems should have similar solutions, the similarity metric plays a critical role in case matching and retrieval. In this paper, we develop a GA-based method for learning the similarity measures of nominal features for CBR classifiers.

Nominal feature is one type of symbolic features whose values are completely unordered. Obviously, the unordered values provide less information than the ordered ones. This is reflected in comparing two cases in a case base. Based on the ordered feature values, we can not only identify whether a given pair of cases has the same feature values or not, but also find out the ordered relation between the two feature values. In contrast, based on the completely unordered feature values, there are only two relationships for a given pair of cases: same or different. From the point of view of information system, these unordered domain values of nominal features lead to coarse information granules, which may bring the difficulty of determining an accurate similarity measure in case matching and retrieval.

There are various forms of distance metrics and similarity measures for different types of features. The most common metrics are Euclidean Distance, Hamming distance, and Cosine coefficients. Among them the Hamming metric is the most often used for nominal features, where the similarity of two different nominal feature values 
is defined as zero and the similarity of two equal feature values is one. To deal with both numerical and symbolic features, many researchers proposed various heterogonous similarity measures [3-6]. However, in their definitions, the similarity measure of the nominal features is still the same to that in Hamming metric. In this research, we make a first attempt to show that, when two nominal feature values are different, the similarity should not always be set as zero. The domain of the similarity will be extended from $\{0,1\}$ to $[0,1]$ to obtain a fine-grained measure of nominal features.

A GA-based supervised learning approach is developed to obtain the similarity measure of nominal values for a given classification problem. Here we assume that there are only limited feature values in the domains of each nominal feature. Theoretically, if there are $n$ elements in the domain of a feature, $n \cdot(n-1) / 2$ similarity values need to be learned to determine the similarity measure of this feature. In practice, it is not necessary to determine so many similarity values. If two different nominal feature values certainly lead to different class labels, the similarity between these two nominal values is assumed as zero. The GA-based method is then used to learn the similarity values of other nominal feature values. The learned similarity values improve the classification accuracy and can be used to analyze the importance of each feature in the given CBR classifier.

\section{Significance of Learning Nominal Feature Similarity: An Illustrative Example}

Table 1 describes a case base with nominal features. The classification problem is to predict which continent a person comes from depending on his hair color and complexion. The features "Hair_Color" $(\boldsymbol{H})$ and "Complexion" $(\boldsymbol{C})$ are conditional features; and the last feature, "Place", is the class label. The domains of the two conditional features are represented by $D_{H}=\{B l, B r, G, R\}$ and $D_{C}=\{Y e, B l, W\}$, respectively.

Table 1. A case base with nominal features

Table 2. Testing cases

\begin{tabular}{|c|c|c|c|}
\hline \hline ID & $\boldsymbol{H}$ & $\boldsymbol{C}$ & Place \\
\hline 1 & $R$ & $B l$ & Asia \\
\hline 2 & $R$ & $Y e$ & Asia \\
\hline 3 & $B l$ & $W$ & Europe \\
\hline \hline
\end{tabular}

\begin{tabular}{|c|c|c|c|}
\hline \hline ID & $\boldsymbol{H}$ & $\boldsymbol{C}$ & Place \\
\hline 1 & $B l$ & $Y e$ & Asia \\
\hline 2 & $B r$ & $B l$ & Asia \\
\hline 3 & $G$ & $Y e$ & Asia \\
\hline 4 & $G$ & $W$ & Europe \\
\hline 5 & $B r$ & $W$ & Europe \\
\hline 6 & $R$ & $W$ & Europe \\
\hline \hline
\end{tabular}

Traditionally, the similarity between every two different nominal feature values is zero. However, from the class labels of the cases in Table 1, $\mathrm{Ye}$ and $\mathrm{Bl}$ should be more similar than $Y e$ and $W$ does. This is based on the observation that either a person has 
the complexion of $\mathrm{Ye}$ or $\mathrm{Bl}$, he comes from Asia; while if the person has $W$ complexion, he surely comes from Europe. If we still assume that the similarity of $Y e$ and $B l$ is zero, problems will arise when classifying new problems (unseen cases). Given a problem $e, e=\{\boldsymbol{H}=R, \boldsymbol{C}=B l\}$, based on the similarity metric in [6], cases 2 and 6 are retrieved with $\operatorname{sim}(e, 2)=\operatorname{sim}(e, 6)=0.5$. Therefore, $e$ cannot be classified to "Asia" or "Europe".

To address the problem, we can adapt the similarity measure of $Y e, B l$, and $W$ for the feature $\boldsymbol{C}$ by making use of the hidden information in the class labels. For example, if we redefine that $\operatorname{sim}(Y e, B l)=1>0$, then the retrieved cases will be 1,2 , 3 , and 6. Based on the majority voting rule, the class label of the unseen case $e$ is determined as "Asia".

This redefined similarity measure can be also used to analyze the goodness of each nominal feature. In this paper, the contribution of a feature to the given classification problem is evaluated by the inconsistent degree caused by using the feature for case retrieval. The smaller the degree, the more useful is the feature. The inconsistency arises when cases have the same feature values but belong to different classes. In this sense, the best two situations (when the inconsistency degree $=0$ ) of a feature $f$ are (i) all cases with different feature values are certainly classified to different classes; (ii) all cases with different feature values belong to the same classes. In the first situation, all the similarities of different feature values of $f$ are equal to zero, and in the second situation, they are equal to one. Therefore, a feature is said to be more useful when its learned similarity values are closer to zero or one. We define a distance $d$ between the similarity values of a feature $f$ and the set $\{0,1\}$ as

$$
d\left(\left\{s_{1}, s_{2}, \ldots, s_{\mathrm{L}}\right\},\{0,1\}\right)=\sum_{i=1}^{L} \min \left\{s_{i}, 1-s_{i}\right\},
$$

where $\left\{s_{1}, s_{2}, \ldots, s_{\mathrm{L}}\right\}$ is the learned set of similarity values of $f$. In the following sections, $d$ is also denoted as $d(f,\{0,1\})$.

\section{Using GA to Learn Similarity Measure for Nominal Features}

This section describes a supervised GA algorithm for learning the similarity values falling in $[0,1]$ instead of $\{0,1\}$. Let there be a case base consisting of $N$ cases $e_{1}$, $e_{2}, \ldots, e_{N}, m$ nominal features $f_{1}, f_{2}, \ldots, f_{m}$. The domain of each feature has limited elements represented by $D_{i}=\left\{v_{i 1}, v_{i 2}, \ldots, v_{m, l_{i}}\right\}, i=1,2, \ldots, m ; v_{i j}$ is a nominal value, $l_{i}$ is the number of different values of the $i$-th feature. As we have mentioned in Section $1, L_{i}=l_{i} \cdot\left(l_{i}-1\right) / 2$ similarity values should be learned at most for the $i$-th feature.

\section{Encoding rule}

In the GA algorithm, each chromosome is encoded as a string consisting of $m$ parts corresponding to the $m$ features. A chromosome $c$ takes the form shown in Fig. 1. For the $i$-th part, there are $L_{i}$ genes represented by $L_{i}$ decimals: $s_{i p} \in[0,1],\left(1 \leq p \leq L_{i}\right)$, representing the similarity measure for the $i$-th feature. The initial values of $s_{i j}(1 \leq p \leq$ $L_{i}$ ) is randomly generated for $i=1,2, \ldots, m$. 


\begin{tabular}{|c|c|c|c|c|c|c|c|c|c|c|c|c|c|c|}
\hline$s_{11}$ & $s_{12}$ & $\cdots$ & $s_{1 L_{1}}$ & & $s_{21}$ & $\cdots$ & $s_{2 L_{2}}$ & & $\cdots$ & & $s_{m 1}$ & $s_{m 2}$ & $\ldots$ & $s_{m L_{m}}$ \\
\hline 0.25 & 0.10 & $\ldots$ & 0.83 & & 0.62 & $\ldots$ & 0.35 & & $\ldots$ & 0.74 & 0.40 & $\ldots$ & 0.56 \\
\hline
\end{tabular}

Fig. 1. A chromosome $c$

\section{Fitness Function}

In this research, the fitness function of a chromosome $c$ is the corresponding classification accuracy using the similarity values indicated in $c$. The classification accuracy is the ratio of the number of correctly classified cases, $n_{c}$, over the whole number of unseen cases, $N_{\mathrm{P}}$. The fitness function is then defined as: fitness $(c)=n_{c} / N_{\mathrm{P}}$.

\section{The GA Algorithm}

a) Initialize the population of the chromosomes. A population set is represented by $\left\{c_{1}, c_{2}, \ldots, c_{P}\right\}$, where $P$ is the size of the population. Each chromosome is encoded as in Fig. 1. Each gene is a randomly initialized to be a decimal in [0, 1], representing the similarity value between two nominal values in the domain of each nominal feature.

b) Selection and crossover. Here the selection probability is set as 1 and the whole set of population is considered to be the mating pool. These settings let the modal to be closer to a random search. In each generation, two chromosomes are randomly selected to perform crossover. The cutting point for crossover is randomly generated and the genes in the two chromosomes that lie behind the cutting point are exchanged to produce an offspring.

c) Mutation. Let the mutation probability be $p_{\text {muta }}$. Randomly select one gene $g$ (with value $v_{\mathrm{g}}$ ) in the newly generated offspring string, and convert the value $v_{\mathrm{g}}$ to $\left(1-v_{\mathrm{g}}\right)$.

d) End condition. Repeat (a)-(c) until the number of generations attains a predefined threshold.

\section{Simulation Results and Analysis}

Two examples are used in the simulations to show the effectiveness of learning similarity measure using the GA algorithm. The used data sets include the example case base in Table 1 and the Balloons database from the UCI repository [7].

\subsection{Example 1}

The cases in Table 1 are used as training data, and those in Table 2 are used as testing cases. There are totally 6 similarity values which need to be determined, five for the feature $\boldsymbol{H}$ and one for the feature $\boldsymbol{C}$. Therefore, each chromosome has two parts, the first of which consists of 5 genes and the second part has only one gene. Here the size of population $P=10$, the terminal number of generations is from 100 to 20000, and the mutation probability $P_{m}=0.05$.

Table 3 shows the results of the learned similarity measure for the nominal features. For feature $\boldsymbol{H}, B l$ and $B r$ is the most similar feature values, and then comes 
Table 3. Learned similarity measure of nominal features (testing accuracy $=1$ )

\begin{tabular}{|c|c|c|c|c|c|c|}
\hline \hline $\begin{array}{l}\text { Number of } \\
\text { Generations }\end{array}$ & $\begin{array}{c}\operatorname{sim}(B l, \\
B r)\end{array}$ & $\begin{array}{c}\operatorname{sim}(B l, \\
G)\end{array}$ & $\begin{array}{c}\operatorname{sim}(B r, \\
G)\end{array}$ & $\operatorname{sim}(G, R)$ & $\begin{array}{c}\operatorname{sim}(B r, \\
R)\end{array}$ & $\begin{array}{c}\operatorname{sim}(Y e, \\
B l)\end{array}$ \\
\hline 100 & 0.38 & 0.07 & 0.29 & 0.74 & 0.19 & 0.45 \\
\hline 500 & 0.69 & 0.72 & 0.53 & 0.45 & 0.50 & 0.55 \\
\hline 1,000 & 0.66 & 0.55 & 0.50 & 0.44 & 0.46 & 0.54 \\
\hline 5,000 & 0.72 & 0.49 & 0.49 & 0.52 & 0.47 & 0.51 \\
\hline 10,000 & 0.76 & 0.50 & 0.50 & 0.50 & 0.51 & 0.50 \\
\hline 20,000 & 0.84 & 0.49 & 0.49 & 0.51 & 0.50 & 0.50 \\
\hline Avg. & 0.68 & 0.47 & 0.47 & 0.53 & 0.44 & 0.51 \\
\hline$d\left(^{*},\{0,1\}\right)$ & 0.36 & \multicolumn{3}{l}{0.16} & \\
\hline \hline
\end{tabular}

the pair of $G$ and $R$; and for feature $C, \operatorname{sim}(Y e, B l)=0.51$ is much greater than $\operatorname{sim}(Y e, W)=\operatorname{sim}(B l, W)=0$. With these similarity values, all the testing cases in Table 2 can be correctly classified by the training cases.

Here $d\left(^{*},\{0,1\}\right)$ in Table 3 denotes the distance of the learned similarities to the set of $\{0,1\}$ defined by Equation 1 , where * means the feature $\boldsymbol{H}$ or $\boldsymbol{C}$. Therefore, the feature $\boldsymbol{C}$ is more important for classifying unseen cases than the feature $\boldsymbol{H}$ does.

\subsection{Example 2}

The Balloons Database consists of 16 cases and 4 nominal features. There are two nominal values in the domain of each conditional feature. It is found that there are 4 similarity values which need to be learned.

Six cases are firstly selected as the training data and the remaining 10 cases are used as testing cases. The original classification accuracy based on the majority voting principle is 0.70 . In the learning process of the GA algorithm, the mutation probability is also set as 0.05 as in Section 4.1.

Table 4. Learned similarity values on Balloons Database (Original accuracy $=0.70$ )

\begin{tabular}{|c|c|c|c|c|c|}
\hline \hline $\begin{array}{c}\text { Number of } \\
\text { Generations }\end{array}$ & $\begin{array}{c}\text { sim(Yellow, } \\
\text { Purple })\end{array}$ & $\begin{array}{c}\text { sim(Small, } \\
\text { Large })\end{array}$ & $\begin{array}{c}\text { sim(Stretch, } \\
\text { Dip })\end{array}$ & $\begin{array}{c}\text { sim(Adult, } \\
\text { Child })\end{array}$ & Accuracy \\
\hline 100 & 0.55 & 0.43 & 0.69 & 0.28 & 1.0 \\
\hline 500 & 0.56 & 0.86 & 0.83 & 0.38 & 0.9 \\
\hline 1,000 & 0.57 & 0.55 & 0.79 & 0.24 & 0.9 \\
\hline 5,000 & 0.63 & 0.51 & 0.29 & 0.03 & 1.0 \\
\hline 10,000 & 0.65 & 0.31 & 0.84 & 0.25 & 1.0 \\
\hline 20,000 & 0.70 & 0.33 & 0.79 & 0.23 & 1.0 \\
\hline Avg. & 0.61 & 0.50 & 0.71 & 0.24 & 0.97 \\
\hline$d\left(^{*},\{0,1\}\right)$ & 0.39 & 0.36 & 0.23 & 0.23 & - \\
\hline \hline
\end{tabular}

Note: * denotes the features, Color, Size, Act, and Age, respectively.

Table 4 shows the learned similarity values for the four nominal features. With these similarity values, the accuracy increases from the original 0.70 to 0.97 . The distances of similarity values to $\{0,1\}$ for "Act" and "Age" are the smallest compared 
with that of other features. Therefore, the features "Act" and "Age" are the most critical features to make the classification decisions. In fact, using only these two features, all the testing cases can be correctly classified based on the majority voting principle. In contrast, with the other two features "Color" and "Size", five out of ten cases are classified to the wrong classes.

\section{Conclusions}

In this paper, we make a first attempt to learn the similarity measures of nominal features using a GA-based approach. Two examples are used to illustrate the effectiveness of the developed learning method. The simulation results show that the testing accuracy increases and the importance of each feature is reflected in the learned similarity values. To summarize, the main contributions are as follows: (i) the similarity between the nominal features has been extended from $\{0,1\}$ to $[0,1]$, which can make the best out of the available information; (ii) this GA-based method is an alternative way to improve the classification accuracy; (iii) based on the learned similarity values, we can further analyze the importance of each nominal feature which can provide potential useful information to enhance the understanding of the data sets.

\section{Acknowledgement}

This work is supported by the CERG research grant \#BQ-496.

\section{References}

1. J. Kolodner. Case-Based Reasoning. Morgan Kaufmann, San Francisco, 1993.

2. S. K. Pal and S. C. K. Shiu. Foundations of Soft Case-Based Reasoning. John Wiley, New York, 2004.

3. D. W. Aha, K. Dennis, and K. A. Marc. Instance-Based Learning Algorithms. Machine Learning, Vol. 6, pp. 37-66, 1991.

4. D. W. Aha. Tolerating noisy, irrelevant and novel attributes in instance-based learning algorithms. International Journal of Man-Machine Studies, Vol. 36, pp. 267-287, 1992.

5. D. R. Wilson and T. R. Martinez. Improved Heterogeneous Distance Functions. Journal of Artificial Intelligence Research, Vol. 6, pages 1-34, 1997.

6. K.C. Gowda and E. Diday. Symbolic clustering using a new similarity measure. IEEE trans. Systems, man Cybernetics, Vol. 22, pp.368-378, 1992.

7. S. Hettich, C. L. Blake, and C. J. Merz. UCI Repository of Machine Learning Databases: [http://www.ics.uci.edu/ mlearn/MLRepository.html]. Irvine, CA: University of California, Department of Information and Computer Science. 